\title{
Sense of Humor and General Life Satisfaction in Association with the Biological Effects of Resistance Training in People with Impaired Glucose Tolerance
}

\author{
Elisabeth Hansen ${ }^{1}$, Kjell Terje Gundersen ${ }^{2}$, Sven Svebak ${ }^{3}$ \\ ${ }^{1}$ Department of Health Sciences, Mid Sweden University, Östersund, Sweden \\ ${ }^{2}$ Department of Sport Sciences and Physical Education, Nord University, Levanger, Norway \\ ${ }^{3}$ Department of Neuromedicine, NTNU Norwegian University of Science and Technology, Trondheim, Norway \\ Email: *elisabeth.hansen@miun.se
}

How to cite this paper: Hansen, E., Gundersen, K.T. and Svebak, S. (2017) Sense of Humor and General Life Satisfaction in Association with the Biological Effects of Resistance Training in People with Impaired Glucose Tolerance. Health, 9, 870882.

https://doi.org/10.4236/health.2017.95062

Received: March 20, 2017

Accepted: May 22, 2017

Published: May 25, 2017

Copyright (๑) 2017 by authors and Scientific Research Publishing Inc. This work is licensed under the Creative Commons Attribution International License (CC BY 4.0).

http://creativecommons.org/licenses/by/4.0/

\begin{abstract}
The aim of the study was to investigate associations between psychological and biological changes due to resistance training in people with impaired glucose tolerance (IGT). Subjects were randomized into maximal (MRT) versus endurance resistance training (ERT) groups. Intervention periods lasted four months. All subjects had blood work that suggested IGT at the initial screening. The ERT acted as a wait-list control group when the MRT performed their training. Baseline scores on general life satisfaction (LISAT) and sense of humor (SHQ-6: a positive coping resources) were obtained. Potential differences between groups (types of intervention and intervention versus control) were investigated by analysis of variance (one-way ANOVA). Correlations were calculated in each group to estimate the degree of covariance between biological and psychological changes from pre- to post-intervention by Pearson and Spearman coefficients. Sense of humor tended to be correlated with a positive reduction of insulin following MRT as well as with reduction in body fat following ERT. Differences across the intervention groups in changes from pre- to post-training in biological variables (glucose, insulin, muscle mass, and corrected percentage of fat) as well as psychological variables (general life satisfaction and sense of humor) were not significant, whereas differences in BMI and weight were significant. By investigating the intervention groups separately in comparison to the control group, the MRT revealed significant improvement by reduction in insulin, percentage of fat, BMI and weight. The ERT caused significant improvements for insulin and percentage of fat, while general life satisfaction had a significant negative development.
\end{abstract}




\section{Keywords}

Life Satisfaction, Humor, Resistance Training, Body Composition, IGT

\section{Introduction}

In order to adhere to a physical training programme, an individual often experiences considerable strain, which may hinder motivational resources to cope with exercise demands [1] [2]. Furthermore, research indicates that affect may play an important role in individuals' decisions to exercise [3], and personality as well as related psychological factors has proven to be associated with participation in physical activity and compliance in numerous studies [4] [5] [6]. Additionally, sense of humor is found to be a health-protecting cognitive coping factor [7]. The present study aimed at investigating associations between psychological and biological changes due to resistance training in people with impaired glucose tolerance (IGT).

Individual differences in psychological characteristics may be associated with effects from exercise prevention programmes. Martinsen and colleagues [8] address the lack of studies on dose-response effects of physical activity or exercise on psychological changes in a review. Such differences may reflect differences in the psychological effects of acute life circumstances, which are seen in the subjective appraisal of overall life and positive as well as negative mood variables (ibid.). Thus, behavioural inhibition in adolescents appears to be inversely related to cardiovascular fitness and tolerance for high-intensity exercise. Adolescents report reduced enjoyment as opposed to individuals scoring high on assessment of behavioural activation, where enjoyment appears to be a more likely outcome, as assessed by scores on psychological measures of behavioural activation and inhibition systems of the brain [9].

Proneness to depression, negative effect, impulsivity and health-seeking behaviour are all negatively associated with a propensity for physical exercise [10]. Measures of self-efficacy among adults in coping with life challenges have been strong predictors of participation in endurance as well as resistance activities [11]. Other studies of psychological correlates of physical exercise have focused on perceived stress and positive emotions. Research may, in this way, be a bit contradictory. In general, findings from studies of adults reflect improved coping and well-being after aerobic training [12]. However, resistance training has generally been left out, and aerobic training has been a focus in most of these studies. Individuals with IGT are at risk of developing a more serious illness in the future [13], and physical functioning might decrease or worsen with more severe diabetic symptoms [14] [15]. Resistance training is recognized as an effective strategy in the primary prevention of obesity and in reducing the risk of developing Type 2 diabetes [16] [17]. Therefore, the aim of the present study was to compare maximal training versus endurance resistance training in changes of general life satisfaction and sense of humor following the training/intervention. 
Furthermore, the purpose was to investigate the importance of differences in the biological and psychological consequences of resistance training and to test whether a range of potential psychobiological co-variations as psychological changes from pre- to post-intervention were associated with biological changes. Covariance was tested in relation to changes in blood glucose, insulin, muscle mass, body fat, weight and body mass. In this way, the present study investigated the significance of psychological factors in the efficacy of physical intervention based upon resistance training.

\section{Methods}

\subsection{Subjects}

Eighteen participants volunteered for the study. They were all classified as having impaired glucose tolerance (IGT) at baseline when they took part in a large-scale public health screening study, the Second North-Tröndelag Health Survey (HUNT II), in Norway (https://www.ntnu.edu/hunt/hunt2; Platou, Midthjell, Romundstad \& Hveem, submitted for publication) according to criteria set by the World Health Organization [18]. Initially 2000 subjects were invited, approximately 1100 subjects responded, and of these, 55 individuals were classified with diabetes and 94 individuals with IGT based on the WHO definition. In the at-risk group of 94 individuals, 70 were eligible to participate in an intervention study, and 18 responded positively to participating in the present study. These individuals were randomly assigned to one of two resistance training intervention groups $(\mathrm{N}=18,14$ female and 4 male participants, age 33 - 69). Group 1 included two males (mean age: $47.5 \mathrm{yrs}$ ) and seven females (mean age: $46.5 \mathrm{yrs}$ ). Group 2 also included two males (mean age: $60.5 \mathrm{yrs}$ ) and seven females (mean age: 44.4 yrs). Participants in both groups (9 individuals, respectively) fulfilled the criterion for overweight according to body mass index (BMI) (Group 1: $\mathrm{M}=28.55, \mathrm{SD}=4.3$; Group 2: $\mathrm{M}=27.17, \mathrm{SD}=4.1$ ). All participants were classified as sedentary and had not engaged in regular physical activity for at least six months prior to the start of the study. Two men were randomized into each group. Although the study did not analyze possible gender differences due to the few men in the study, male participants were included to retain the total number of the population. The present study is part of a larger investigation on several significant parameters of this high-risk population, those with IGT, and the findings of the study might provide an additional interesting perspective on the issues focused on in this paper.

The sociodemographic characteristics of the participants are briefly described in Table 1 . The group is predominantly female, and most of the participants are married/cohabited and employed.

\subsection{Procedure}

Group 1 engaged in supervised maximal resistance training (MRT: Bernstein inverted pyramid system: $5 \times 3-5 \times 4,60 \%-85 \% 1 \mathrm{RM}$ ) [19] three days/week over four months. During this intervention period, the members of Group 2 
Table 1. Sociodemographic descriptive data of the study group $(\mathrm{N}=18)$.

\begin{tabular}{cc} 
Gender (N =18) & 4 \\
Men & 14 \\
Women & \\
Age (Mean Age) & 54.0 \\
Men & 45.5 \\
Women & \\
Civil Status (N = 18) & 3 \\
Single/Widow & 15 \\
Married/Cohabited & \\
Occupational Position (N = 18) & 15 \\
Employed & 3 \\
Unemployed & \\
Education Level (N = 18) & 5 \\
Lower Secondary School & 11 \\
Higher Secondary School & 2 \\
University (3 years or more) & \\
\hline
\end{tabular}

acted as waiting-list control subjects. Later, Group 2 engaged in supervised endurance resistance training (ERT: $3 \times 12-3 \times 15,45 \%-65 \% 1 \mathrm{RM}$ ) [20] three days/week over a four month period, with the two pre-baseline assessments as reference measures. Both interventions consisted of eight exercises that involved the entire skeletal muscular system of the body. During the second intervention (Group 2) consisting of conventional ERT, there were two drop-outs. Mean values then replaced the missing values.

\section{Assessment of biological variables}

Standard procedure for venous blood samples, with laboratory estimates of serum levels of insulin and fasting glucose, were applied before and after intervention and before the waiting list period (Group 2). Serum samples were analysed at the Central Laboratory of Levanger Hospital on an Abbot Architect ci8200 auto analyser. Glucose levels were measured using a hexokinase G-6TDH method. Serum levels of insulin were determined at the Hormone Laboratory at Oslo University Hospital at Aker using DELFIA kits form PerkinElmer Life Sciences, Wallac Oy, Turku, Finland.

Clinical measures were taken with the participants wearing undergarments without shoes, and height and weight were adopted for the BMI algorithm $\left(\mathrm{kg} / \mathrm{m}^{2}\right)$. The WHO categories for BMI were used [21]: Normal weight (18.5 $24)$, overweight (25 - 29) and obese ( $\geq 30)$. Furthermore, waist circumference was measured horizontally at the height of the umbilicus to the nearest $1 \mathrm{~cm}$ when the participant was standing with their arms hanging relaxed. Skin-fold measures on biceps, triceps, subscapularis and suprailiac were included in the algorithm to calculate total body fat index, controlled for age, gender and waist circumference [22].

Upper leg skeletal muscle mass was estimated by magnetic resonance imaging (MRI) technology by use of a $1.5 \mathrm{~T}$ GE Signa HDX MRI scan with software version 14.0.M5. The measurement protocol was based on T1 weighted FSE (Fast Spin Ecco) MRI in order to be able to make an image suitable for measuring the 
parameters of interest. This measurement was found to be sufficient in order to clearly differentiate between muscle mass, subcutaneous adipose tissue and bone tissue. More details for the assessment of the biological and clinical characteristics are given by Hansen and colleagues [23].

\subsection{Assessment of Psychological Characteristics and Reliability Tests}

The participants completed questionnaires to assess their psychological characteristics. The scales included general life satisfaction and sense of humor (a positive coping mechanism). General life satisfaction was scored by use of the 11-item LISAT scale [24] according to a six-step format (very dissatisfying to very satisfying), as illustrated by item no. 1: "Life as a whole is" (Chronbach's alpha $(\mathrm{CA})=0.84)$.

Sense of humor was assessed by a six-item scale (Sense of Humor Questionnaire-6, SHQ-6: Svebak, 1996, 2010), where a four-step scoring format was applied (very much to very little), as illustrated by item 5: "Would you say that you have much cause for amusement during an ordinary day?" (Chronbach's alpha $(C A)=0.60)$. Changes in self-reported general life satisfaction and sense of humor were estimated as the differences from pre- to post-intervention in all subjects.

\subsection{Data Scoring and Statistical Analyses}

Pre- and post-assessments of biological and psychological variables identified a magnitude of change induced by training, on the assumption that training increases the likelihood of having a positive difference score (delta value). Thus, for fasting glucose levels, the post-value vas subtracted from the pre-value on the assumption that training would cause reduced fasting glucose levels. Also, for blood insulin levels, the post-value was subtracted from the pre-value.

For skeletal muscle mass, training was predicted to increase muscle mass; therefore, the pre-value was subtracted from the post-value. Percent of body fat (corrected for age and gender) was also investigated. Training was predicted to reduce fat; therefore, post-values were subtracted from pre-values. The same procedure of subtraction of post-values from pre-values, was followed for the variables of weight and BMI.

For the psychological variables, differences from pre- to post-intervention were estimated to increase the likelihood of having positive scores, and the pre-intervention value was subtracted from the post-intervention value. High change scores (delta values) for SHQ-6 (sense of humor) and LISAT (general life satisfaction) reflected higher scores after intervention than before.

The SPSS 24.0 statistical package was applied in all statistical analyses (SPSS Inc, Chicago, Ill, USA). Background analysis by frequencies and T-tests were carried out to assess homogeneity between the groups. To test internal consistency, scale reliability between items was performed for the two psychological variables sense of humor $(\mathrm{CA}=0.60)$ and general life satisfaction $(\mathrm{CA}=0.84)$. Coefficients of correlation were calculated in each group to estimate the degree 
of covariance between biological and psychological changes from pre- to post-intervention. Given the low number of subjects, Spearman coefficients were applied, and two- and one-way probability levels for alpha coefficients were calculated. Potential differences between groups (types of intervention and intervention versus control) were investigated by analysis of variance (one-way ANOVA).

\section{Ethics}

Written informed consent was obtained from each of the study participants in accordance with the Helsinki Declaration [25]. The study was approved by the Regional Committee for Ethics in Medical Research (Ref. No. 4.2006.2549).

\section{Results}

Table 2 presents changes in the biological and psychological variables from preto post-intervention. There were significant differences across the two groups (maximal resistance versus endurance resistance) regarding weight (mean value 2.75 vs $-5.35, \mathrm{p}=0.02$ ) and BMI (mean value $1.14 \mathrm{vs}-0.37, \mathrm{p}=0.04$ ). Regarding muscle and insulin levels, the values were nearly significant (Table 2).

Table 3 and Table 4 present results for changes in the biological and psychological variables from pre- to post-intervention (intervention versus control). Table 3 shows the mean difference between the maximal resistance group and the control group. A positive development was observed for insulin levels (mean value 8.00 vs $-4.66, \mathrm{p}=0.03$ ), percentage of fat (mean value 1.04 vs $-1.63, \mathrm{p}=$ 0.00 ), BMI (mean values 1.14 vs $-0.01, \mathrm{p}=0.01$ ), and weight (mean values 2.75 vs $-0.03, \mathrm{p}=0.01)$.

Table 2. Means and 95\% CI for changes from pre to post intervention in blood levels of fasting glucose (glucose), insulin, muscle mass (muscle), Percentage of fat (corr. \% fat), body mass index (BMI), and weight (biological variables) as well as changes in SHQ-6, and LISAT (psychological variables) in the intervention groups (Maximal Resistance: MRT; Endurance Resistance: ERT). F-scores and p-values from One-WAY ANOVA tests are included.

\begin{tabular}{ccccccccc}
\hline & \multicolumn{1}{c}{ MRT $(\mathrm{N}=9) 95 \%$ CI } & \multicolumn{2}{c}{ ERT $(\mathrm{N}=9) 95 \%$ CI } & \\
\hline Variables & Means & Lower & Upper & Means & Lower & Upper & F & p-value \\
\hline Biological Variables & & & & & & & & \\
\hline Glucose (mmol/l) & 0.11 & -0.14 & 0.36 & 0.01 & -0.26 & 0.28 & 0.38 & n.s. \\
Insulin (pmol/l) & 8.00 & -1.31 & 17.31 & 21.22 & 8.76 & 33.67 & 3.84 & $\mathbf{0 . 0 6}$ \\
Muscle & 69.3 & -315.2 & 453.9 & 1809.0 & -274.5 & 3892.5 & 3.6 & $\mathbf{0 . 0 7}$ \\
Corr. \% fat & 1.04 & -0.06 & 2.15 & 2.11 & 0.63 & 3.58 & 1.77 & n.s. \\
BMI & 1.14 & 0.32 & 1.97 & -0.37 & -1.75 & 0.99 & 4.80 & $\underline{\mathbf{0 . 0 4}}$ \\
Weight & 2.75 & 0.87 & 4.64 & -5.35 & -12.84 & 2.13 & 5.86 & $\underline{\mathbf{0 . 0 2}}$ \\
\hline Psychological Variables & & & & & & & & \\
\hline SHQ-6 & 1.22 & -0.20 & 2.64 & 0.44 & -0.89 & 1.78 & 0.84 & n.s. \\
LISAT & -1.11 & -5.97 & 3.75 & -4.88 & -10.30 & 0.52 & 1.43 & n.s. \\
\hline
\end{tabular}

n.s. $=$ not significant. Bold values $=\leq 0.07$, Bold and underlined values $=\leq 0.05 . \mathrm{BMI}=$ body mass index. $\mathrm{SHQ}=$ sense of humor. LISAT $=$ general life satisfaction. 
Table 3. Means and 95\% CI for changes from pre to post intervention in blood levels of fasting glucose (glucose), insulin, muscle mass (muscle), corrected percentage of fat (corr. \% fat), body mass index (BMI), and weight (biological variables) as well as changes in SHQ-6, and LISAT (psychological variables) in the intervention group (Maximal Resistance: MRT) versus control group. F-scores and p-values from One-WAY ANOVA tests are included.

\begin{tabular}{|c|c|c|c|c|c|c|c|c|}
\hline \multirow[b]{2}{*}{ Variables } & \multicolumn{3}{|c|}{ MRT (N = 9) 95\% CI } & \multicolumn{3}{|c|}{ Control $(\mathrm{N}=9) 95 \% \mathrm{CI}$} & \multirow[b]{2}{*}{$\mathrm{F}$} & \multirow[b]{2}{*}{ p-value } \\
\hline & Means & Lower & Upper & Means & Lower & Upper & & \\
\hline \multicolumn{9}{|l|}{ Biological Variables } \\
\hline Glucose $(\mathrm{mmol} / \mathrm{l})$ & 0.11 & -0.14 & 0.36 & 0.00 & -0.19 & 0.19 & 0.63 & n.s. \\
\hline Insulin (pmol/l) & 8.00 & -1.31 & 17.31 & -4.66 & -12.92 & 3.59 & 5.50 & $\underline{0.03}$ \\
\hline Muscle & 69.3 & -315.2 & 453.9 & 8.2 & -522.7 & 539.1 & 0.04 & n.s. \\
\hline Corr. \% fat & 1.04 & -0.06 & 2.15 & -1.63 & -2.97 & -0.29 & 12.63 & $\underline{0.00}$ \\
\hline BMI & 1.14 & 0.32 & 1.97 & -0.01 & -0.46 & 0.42 & 8.23 & $\underline{0.01}$ \\
\hline Weight & 2.75 & 0.87 & 4.64 & -0.03 & -1.18 & 1.12 & 8.46 & $\underline{0.01}$ \\
\hline \multicolumn{9}{|c|}{ Psychological Variables } \\
\hline SHQ-6 & 1.22 & -.20 & 2.64 & 0.00 & -1.80 & 1.80 & 1.50 & n.s. \\
\hline LISAT & -1.11 & -5.97 & 3.75 & 0.22 & -1.52 & 1.97 & 1.35 & n.s. \\
\hline
\end{tabular}

n.s. $=$ not significant. Bold values $=\leq 0.05 . \mathrm{BMI}=$ body mass index. SHQ $=$ sense of humor. LISAT $=$ general life satisfaction.

Table 4. Means and 95\% CI for changes from pre to post intervention in blood levels of fasting glucose (glucose), insulin, muscle mass (muscle), Percentage of fat (corr. \% fat), body mass index (BMI), and weight (biological variables) as well as changes in SHQ-6, and LISAT (psychological variables) in the intervention group (Endurance resistance: ERT) versus control group. F-scores and p-values from One-WAY ANOVA tests are included.

\begin{tabular}{ccccccccc}
\hline & \multicolumn{7}{c}{ ERT $(\mathrm{N}=9) 95 \% \mathrm{CI}$} & \multicolumn{7}{c}{ Control $(\mathrm{N}=9)$} & $95 \% \mathrm{CI}$ & & \\
\hline Variables & Means & Lower & Upper & Means & Lower & Upper & $\mathrm{F}$ & p-value \\
\hline Biological Variables & & & & & & & & \\
\hline Glucose (mmol/l) & 0.11 & -0.25 & 0.27 & 0.00 & -0.19 & 0.19 & 0.01 & n.s. \\
Insulin (pmol/1) & 21.22 & 8.76 & 33.67 & -.4 .66 & -12.92 & 3.59 & 15.95 & $\underline{\mathbf{0 . 0 0}}$ \\
Muscle & 1809.0 & -274.5 & 453.9 & 8.2 & -522.7 & 539.1 & 3.73 & $\mathbf{0 . 0 7}$ \\
Corr. \% fat & 2.11 & 0.63 & 3.58 & -1.63 & -2.97 & -0.29 & 18.77 & $\underline{\mathbf{0 . 0 0}}$ \\
BMI & -0.37 & -1.75 & 0.99 & -0.01 & -0.46 & 0.42 & 0.32 & n.s. \\
Weight & -5.35 & -12.84 & 2.13 & -0.03 & -1.18 & 1.12 & 2.62 & n.s. \\
\hline Psychological Variables & & & & & & & & \\
\hline SHQ-6 & 0.44 & -0.89 & 1.78 & 0.00 & -1.80 & 1.80 & 0.20 & n.s. \\
LISAT & -5.11 & -10.41 & 0.19 & 0.22 & -1.52 & 1.97 & 4.84 & $\underline{\mathbf{0 . 0 4}}$ \\
\hline
\end{tabular}

n.s. $=$ not significant. Bold values $=\leq 0.07$, Bold and underlined values $=\leq 0.05$. BMI $=$ body mass index. $\mathrm{SHQ}=$ sense of humor. LISAT $=$ general life satisfaction.

Table 4 shows the differences between the endurance resistance group and the control group. Additionally, a positive effect was found regarding insulin levels (mean value $21.22 \mathrm{vs}-4.66, \mathrm{p}=0.00$ ) and percentage of fat (mean value 2.11 
Table 5. (A) biological changes from correlations between psychological and biological changes from pre- to post-intervention as well as (B) between scores on psychological changes, in the maximal resistance training group $(\mathrm{N}=9)$.

\begin{tabular}{ccc}
\hline \multicolumn{3}{c}{ Psychological Variables } \\
\hline A) Biological Variables & SHQ-6 diff. & LIASAT diff. \\
\hline Glucose Fasting & & \\
Insulin & $-0.16(-0.04)$ & $0.53(0.55)$ \\
Muscle Mass & $0.58\left(0.67^{\star}\right)$ & $0.22(0.20)$ \\
Corr. \% fat & $0.12(0.15)$ & $0.24(0.26)$ \\
Body mass index & $0.63(0.58)$ & $0.15(-0.00)$ \\
Weight & $0.34(0.39)$ & $0.42(0.55)$ \\
\hline B) Psychological Variables & $0.32(0.24)$ & $0.43(0.40)$ \\
\hline SHQ-6 diff & - & - \\
LISAT diff & $-0.22(-0.04)$ & \\
\hline
\end{tabular}

${ }^{*} \mathrm{p}<0.10 ;{ }^{* *} \mathrm{p}<0.05$ : two-tailed Pearson correlation coefficients, and one-tailed Spearman rank coefficients (in parentheses). Body mass index $=$ BMI. SHQ $=$ sense of humor. LISAT $=$ general life satisfaction.

Table 6. (A) Correlations between psychological and biological as well as clinical changes from pre- to post-intervention, and (B) between scores on psychological changes, in the endurance resistance training group $(\mathrm{N}=9)$.

\begin{tabular}{ccc}
\hline \multicolumn{3}{c}{ Psychological Variables } \\
\hline & SHQ-6 diff. & LIASAT diff. \\
\hline A) Biological Variables & $0.52(-0.43)$ & $-0.25(-0.20)$ \\
\hline Glucose Fasting & $0.15(0.39)$ & $-0.18(-0.06)$ \\
Insulin & $-0.06(0.43)$ & $0.63(0.33)$ \\
Muscle Mass & $0.81^{* *}(0.05)$ & $0.04(0.40)$ \\
Corr. \% fat & $-0.20(-0.44)$ & $-0.42(-0.22)$ \\
Body mass index & $0.02(-0.54)$ & $-0.48(-0.32)$ \\
Weight & - & - \\
\hline B) Psychological variables & $-0.13(-0.16)$ & \\
\hline SHQ-6 diff & & \\
LISAT diff & & \\
\hline
\end{tabular}

${ }^{*} \mathrm{p}<0.10 ;{ }^{* *} \mathrm{p}<0.05$ : two-tailed Pearson correlation coefficients, and one-tailed Spearman rank coefficients (in parentheses). Body mass index $=\mathrm{BMI}$. SHQ $=$ sense of humor. LISAT $=$ general life satisfaction.

vs $-1.63, \mathrm{p}=0.00)$. Additionally, muscle seems to have a tendency $(\mathrm{p}=0.07)$ towards significant differences between the intervention and control groups in favor of the intervention group (Table 4). General life satisfaction seems to develop negatively for the (endurance resistance) intervention group compared to that of the control group. ( -5.11 vs $0.22, \mathrm{p}=0.04)$.

Correlation coefficients shown in Table 5 reflect changes in Group 1 (maximal resistance training), testing the degree of covariance of changes from pre- to post-intervention between psychological and biological variables (A: upper sec- 
tion) and between changes in the psychological variables (B: lower section). For Sense of humor (SHQ-6), a (marginal) covariance for change was seen with the Spearman rank coefficient $(0.67, \mathrm{p}=0.02)$ due to positive reductions in insulin.

Correlation coefficients given in Table 6 reflect changes in Group 2 (endurance resistance training), testing the degree of covariance of changes pre- to post-intervention. The results regarding sense of humor (SHQ-6) correlated with the positive development regarding reduction in percentage of fat, as seen by the Pearson correlation coefficient $(0.81, \mathrm{p}=0.00)$.

\section{Discussion}

The changes for the body composition variables of weight and BMI are significantly different across the two intervention groups in favour of the maximal resistance group. The current study focused on individuals at risk (IGTs) of developing Type 2 diabetes, who are often overweight. These findings are noteworthy compared to existing research, where resistance training is positively connected to prevention of obesity and Type 2 diabetes [16] [17]. The maximal resistance group seems to benefit the most as demonstrated by reductions in insulin, percentage of fat, weight and BMI. The endurance resistance group showed improvements through reductions in insulin and percentage of fat. These results are in line with existing knowledge (ibid.).

General life satisfaction for the endurance resistance group indicated a negative development (Table 4). Improvement in the present biological measures of insulin and percentage of fat should relate positively to changes in general life satisfaction. This could be an example in which physical training programmes present the individual with strain, which may be taxing on motivational resources needed to cope with exercise demands [1] [2] and in this way, develop negatively.

Correlation coefficients indicate positive changes in sense of humor due to a positive reduction in insulin levels in the maximal resistance group (Table 5) and a positive development regarding sense of humor related to the reduction of body fat in the endurance resistance group (Table 6). These findings indicate that the psychological coping variable of sense of humor might be connected to biological changes following resistance training. This is in line with research [7] that has shown that humor was found to be connected to health, protection and coping factors.

General life satisfaction does not correlate with any of the biological variables, and this might be somewhat surprising, reflecting some of the typical positive developments regarding body composition (weight, BMI and percentage of fat) and improvements in insulin reduction. One could argue that these improvements would have been expected to be positively motivated in correlation with general life satisfaction, which is not the case. However, these findings support that adolescents tend to report reduced enjoyment for high-intensity exercise [9]. This reflection is supported by the fact that both groups in the present study had a negative development in life satisfaction after performing the resistance 
training.

Previous studies on the effects of resistance training do not differentiate between maximal and endurance resistance training in the primary prevention of Type 2 diabetes and IGT. Resistance training in general has been compared with the effects of aerobic endurance training.

Type 2 diabetes patients are more likely to be concerned about their health condition and consequences of their metabolic syndrome than are individuals with IGT. Consequently, they may be likely to demonstrate more compliance to a regular physical training regimen than are those in group of individuals at risk for developing diabetes. If so, primary prevention based upon resistance as well as aerobic training may increase the motivation to comply in IGT individuals by presenting promising results from empirical research upon biological as well as psychological changes including increases in quality of life [26] [27], reduction in anxiety and depression, as well as reduced satisfaction with body weight among Type 2 diabetes patients with high BMI [28]. Exercise in general has positive effects on self-appraisal as well as mood [29]. In the current study, resistance training proved to be efficient in reducing BMI [30] as well as increasing health related quality of life [31]. The present results suggest that the sense of humor may be a positive mental resource to cope with the strain of compliance in resistance training for the primary prevention of IGT.

\section{Conclusion}

The beneficial changes in sense of humor were the only psychological variable that yielded any significant correlation and appeared to be more strongly associated with the effects of endurance resistance training than with the effects of maximal resistance training. Improvements in biological parameters were present in both intervention groups, and the maximal resistance group showed the highest level of benefits. The results of the present study are slightly contradictory, and further studies, including larger populations in large-scale interventions, are needed to verify any psychological association with biological effects of resistance training in people with IGT.

\section{Limitations and Strength of the Study}

The primary strength of the current study is related to the project, the content of the two intervention programmes, how thoroughly the programmes were continuously planned and carried out during the intervention periods and the quality of objective biological tests as well as subjective psychological measures. The resistance training interventions were not self-administered. The subjects were supervised individually and closely followed during their exercise periods by professional and experienced trainers working with clients with diabetes as well as healthy individuals at the work-out centre. Consequently, as expected, the final drop-out was extremely low (2 subjects). Testing procedures and standards were followed carefully, including all biological parameters. Furthermore, two subjective psychological measures were based on validated international scales. 
However, the results should be treated with caution, as they may be influenced by a relatively low number of subjects included in the study. More studies are needed to test the covariance of biological and psychological changes due to resistance training in people with IGT. Traditionally, most of the research focus has been on aerobic exercise; thus, this study might provide a contribution to the research field by increased attention towards resistance exercise.

\section{Acknowledgements}

The participants are acknowledged for their willingness to take part in the project.

\section{Conflict of Interest}

There are no conflicts of interest among the authors. The project was supported by grants from the EU-program Interreg III A, Nord Tröndelag University College, Norway, and Mid Sweden University.

\section{References}

[1] Jallinoja, P., Pajari, P. and Absetz, P. (2008) Repertoires of Lifestyle Change and Self-Responsibility among Participants in an Intervention to Prevent Type 2 Diabetes. Scandinavian Journal of Caring Sciences, 22, 455-462.

https://doi.org/10.1111/j.1471-6712.2007.00551.x

[2] Hansen, E., Landstad, B.J., Hellzen, O. and Svebak, S. (2011) Motivation for Lifestyle Changes to Improve Health in People with Impaired Glucose Tolerance. Scandinavian Journal of Caring Sciences, 25, 484-490. https://doi.org/10.1111/j.1471-6712.2010.00853.x

[3] Catellier, J.R.A. and Yang, Z.J. (2013) The Role of Affect in the Decision to Exercise: Does Being Happy Lead to a More Active Lifestyle? Psychology of Sport and Exercise, 14, 275-282.

[4] Kehler, D., Christensen, B., Lauritzen, T., Christensen, M.B., Edwards, A. and Risor, M.B. (2008) Ambivalence Related to Potential Lifestyle Changes Following Preventive Cardiovascular Consultations in General Practice: A Qualitative Study. BMC Family Practice, 9, 50. https://doi.org/10.1186/1471-2296-9-50

[5] Snellman, I. and Wikblad, K. (2006) Health in Patients with Type 2 Diabetes: An Interview Study Based on the Welfare Theory of Health. Scandinavian Journal of Caring Sciences, 20, 462-471. https://doi.org/10.1111/j.1471-6712.2006.00447.x

[6] Lawton, J., Ahmad, N., Hanna, L., Douglas, M. and Hallowell, N. (2006) “I Can’t Do Any Serious Exercise": Barriers to Physical Activity amongst People of Pakistani and Indian Origin with Type 2 Diabetes. Health Education Research, 21, 43-54. https://doi.org/10.1093/her/cyh042

[7] Romundstad, S., Svebak, S., Holen, A. and Holmen, J. (2016) A 15-Year Follow-Up Study of Sense of Humor and Causes of Mortality: The Nord-Trøndelag Health Study. Psychosomatic Medicine, 78, 345-353.

https://doi.org/10.1097/PSY.0000000000000275

[8] Martinsen, E.W. (2008) Physical Activity in the Prevention and Treatment of Anxiety and Depression. Nordic Journal of Psychiatry, 62, 25-29.

https://doi.org/10.1080/08039480802315640

[9] Schneider, M.L. and Graham, D.J. (2009) Personality, Physical Fitness, and Affec- 
tive Response to Exercise among Adolescents. Medicine \& Science in Sports \& EXercise, 41, 947-955.

[10] Palomo, T., Beninger, R.J., Kostrzewa, R.M. and Archer, T. (2008) Affective Status in Relation to Impulsive, Motor and Motivational Symptoms: Personality, Development and Physical Exercise. Neurotoxicity Research, 14, 151-168. https://doi.org/10.1007/BF03033807

[11] Plotnikoff, R.C., Trinh, L., Courneya, K.S., Karunamuni, N. and Sigal, R.J. (2011) Predictors of Physical Activity in Adults with Type 2 Diabetes. American Journal of Health Behavior, 35, 359-370.

[12] Dyer 3rd, J.B. and Crouch, J.G. (1988) Effects of Running and Other Activities on Moods. Perceptual and Motor Skills, 67, 43-50.

https://doi.org/10.2466/pms.1988.67.1.43

[13] Hansen, E., Landstad, B.J., Gundersen, K.T. and Svebak, S. (2012) The Relative Importance of Aerobic Capacity, Physical Activity and Body Mass Index in Impaired Glucose Tolerance and Type 2 Diabetes. Society, Health \& Vulnerability, 3.

[14] Tapp, R.J., O’Neil, A., Shaw, J.E., Zimmet, P.Z. and Oldenburg, B.F., on behalf of the AusDiab Study Group (2010) Is There a Link between Components of HealthRelated Functioning and Incident Impaired Glucose Metabolism and Type 2 Diabetes? The Australian Diabetes Obesity and Lifestyle (AusDiab) Study. Diabetes Care, 33, 757-762. https://doi.org/10.2337/dc09-1107

[15] Weyer, C., Tataranni, P.A., Bogardus, C. and Pratley, R.E. (2001) Insulin Resistance and Insulin Secretory Dysfunction Are Independent Predictors of Worsening of Glucose Tolerance during Each Stage of Type 2 Diabetes Development. Diabetes Care, 24, 89-94. https://doi.org/10.2337/diacare.24.1.89

[16] Colberg, S.R., Sigal, R.J., Fernhall, B., Regensteiner, J.G., Blissmer, B.J., Rubin, R.R., Chasan-Taber, L., Albright, A.L. and Braun, B. (2010) Exercise and Type 2 Diabetes. The American College of Sports Medicine and the American Diabetes Association: Joint Position Statement. Diabetes Care, 33, e147-e167. https://doi.org/10.2337/dc10-9990

[17] Gram, B., Holtermann, A., Søgaard, K. and Sjøgaard, G. (2012) Effect of Individualized Worksite Exercise Training on Aerobic Capacity and Muscle Strength among Construction Workers-A Randomized Controlled Intervention Study. Scandinavian Journal of Work, Environment \& Health, 38, 467-475.

[18] WHO (1999) Definition, Diagnosis and Classification of Diabetes Mellitus and Its Complications: Report of a WHO Consultation. Part 1, Diagnosis and Classification of Diabetes Mellitus. World Health Organization, Department of Noncommunicable Disease Surveillance, Geneva.

[19] Bernstein, R.K. (2011) Dr. Bernstein's Diabetes Solution: The Complete Guide to Achieving Normal Blood Sugars. Newly rev. and updated ed., Little, Brown, Boston, MA; London.

[20] Black, L.E., Swan, P.D. and Alvar, B.A. (2010) Effects of Intensity and Volume on Insulin Sensitivity during Acute Bouts of Resistance Training. The Journal of Strength and Conditioning Research, 24, 1109-1116.

[21] WHO (2000) Obesity: Preventing and Managing the Global Epidemic. Report of a WHO Consultation, Geneva.

[22] Durnin, J.V. and Womersley, J. (1973) Total Body Fat, Calculated from Body Density, and Its Relationship to Skinfold Thickness in 571 People Aged 12 - 72 Years. Proceedings of the Nutrition Society, 32, 45A.

[23] Hansen, E., Landstad, B.J., Gundersen, K.T., Torjesen, P.A. and Svebak, S. (2012) 
Insulin Sensitivity after Maximal and Endurance Resistance Training. The Journal of Strength and Conditioning Research, 26, 327-334. https://doi.org/10.1519/JSC.0b013e318220e70f

[24] Fugl-Meyer, A.R., Melin, R. and Fugl-Meyer, K.S. (2002) Life Satisfaction in 18- to 64-Year-Old Swedes: In Relation to Gender, Age, Partner and Immigrant Status. Journal of Rehabilitation Medicine, 34, 239-246. https://doi.org/10.1080/165019702760279242

[25] World Medical Association. Declaration of Helsinki (2008) Ethical Principles for Medical Research Involving Human Subjects. http://www.naho.ca/documents/it/2008-WMA-Declaration-Helsinki.pdf

[26] Nicolucci, A., Balducci, S., Cardelli, P., Cavallo, S., Fallucca, S., Bazuro, A., Simonelli, P., Iacobini, C., Zanuso, S. and Pugliese, G. (2012) Relationship of Exercise Volume to Improvements of Quality of Life with Supervised Exercise Training in $\mathrm{Pa}$ tients with Type 2 Diabetes in a Randomised Controlled Trial: The Italian Diabetes and Exercise Study (IDES). Diabetologia, 55, 579-588.

https://doi.org/10.1007/s00125-011-2425-9

[27] Benton, M.J. and Schlairet, M.C. (2012) Improvements in Quality of Life in Women after Resistance Training Are Not Associated with Age. Journal of Women \& Aging, 24, 59-69. https://doi.org/10.1080/08952841.2012.638877

[28] Noor, S.Z., Lua, P.L. and Nik, M.M. (2011) Body Weight Satisfaction: Association with Weight Control Practices among Type 2 Diabetic Patients. Malaysian Journal of Nutrition, 17, 55-66.

[29] Annesi, J.J. and Unruh, J.L. (2008) Relations of Exercise, Self-Appraisal, Mood Changes and Weight Loss in Obese Women: Testing Propositions Based on Baker and Brownell's (2000) Model. The American Journal of the Medical Sciences, 335, 198-204. https://doi.org/10.1097/MAJ.0b013e318152010c

[30] Hansen, E., Landstad, B.J., Brønn, R., Gundersen, K.T. and Svebak, S. (2011) Exercise-Induced Changes in Body Fat, Upper Leg Skeletal Muscle Area, BMI and Body Weight in Overweight People with Risk of Developing Type 2 Diabetes. Acta Kinesiologiae Universitatis Tartuensis, 17, 66-79. https://doi.org/10.12697/akut.2011.17.05

[31] Hansen, E., Vinberg, S., Gundersen, K.T. and Landstad, B.J. (2016) Resistance Training in People at Risk of Developing Type 2 Diabetes and Their Experience of Health-Related Quality of Life. Health, 8, 1323-1334.

https://doi.org/10.4236/health.2016.813133 
Submit or recommend next manuscript to SCIRP and we will provide best service for you:

Accepting pre-submission inquiries through Email, Facebook, LinkedIn, Twitter, etc. A wide selection of journals (inclusive of 9 subjects, more than 200 journals)

Providing 24-hour high-quality service

User-friendly online submission system

Fair and swift peer-review system

Efficient typesetting and proofreading procedure

Display of the result of downloads and visits, as well as the number of cited articles Maximum dissemination of your research work

Submit your manuscript at: http://papersubmission.scirp.org/

Or contact health@scirp.org 